\title{
COORDINATIZATION IN SUPERSTABLE THEORIES. II
}

\author{
STEVEN BUECHLER
}

\begin{abstract}
In this paper we prove
THEOREM A. Suppose that $T$ is superstable and $U(a / A)=\alpha+1$, for some $\alpha$. Then in $T^{\mathrm{eq}}$ there is a $c \in \operatorname{acl}(A a) \backslash \operatorname{acl}(A)$ such that one of the following holds.

(i) $U(c / A)=1$.

(ii) $\operatorname{stp}(c / A)$ has finite Morley rank. In fact, this strong type is semiminimal with respect to a strongly minimal set.
\end{abstract}

0. Introduction. While we gave some motivation for these "coordinatization theorems" in $\S 2$ of $[\mathbf{C I}]$, we would like to give another outline, emphasizing different aspects. The starting point, as always, is [CHL, 4.1]

COORDinatization ThEOREM 0.1. Suppose that $T$ is $\omega$-categorical, $\omega$-stable and $P$ is a 0-definable atom. Then in $T^{\mathrm{eq}}$ there is a 0-definable atom $A$ of Morley rank 1 such that for all $a \in P, \operatorname{acl}(a) \cap A \neq \varnothing$.

In applications this theorem is usually iterated in the following manner. Let $\operatorname{crd}(a)=\operatorname{acl}(a) \cap A$. Define an equivalence relation $\sim$ on $P$ by $a \sim b$ iff $\operatorname{crd}(a)=$ $\operatorname{crd}(b)$. Let $c \in C^{\mathrm{eq}}$ be $a / \sim$. Then $t(a / c)$ is an atom, so we can coordinatize it with a $c$-definable rank 1 atom. (See the notion of a filtration in [B5 and $\mathbf{A}]$.) The version of the Coordinatization Theorem which most readily lends itself to this iteration process is

COORDinatization TheOREM 0.2 . Let $T$ and $P$ be as above. Then in $T^{\mathrm{eq}}$ there is a 0-definable atom $A$ of Morley rank 1 such that for all $a \in P$ there is a $c \in \operatorname{dcl}(a) \backslash \operatorname{acl}(\phi)$ with $c \in \operatorname{acl}(A)($ in fact,$c \in \operatorname{acl}(\operatorname{acl}(c) \cap A))$.

We motivated the results in $[\mathbf{C I}]$ by saying that we simply generalize the proofs of the above theorems to the superstable context. Admittedly, this is not very satisfying. There is no theorem in [CI] which looks very much like one of the above. So, let us forget about proofs for the moment and look for theorems in the superstable setting which look like one of the above. Of course, the first place to look is in $[\mathbf{S h}]$. We find a somewhat similar result involving the following concept. (Recall that $q$ is minimal if it is stationary and $U(q)=1$.)

Definition. Let $A$ and $D_{0}$ be sets and $q_{0} \in S\left(D_{0}\right)$ be minimal. We say that $\operatorname{stp}(a / A)$ is $q_{0}$-semiminimal if there are: $c_{0}$ realizing $q_{0}$ and for $i \leq n$ (some $n$ ), $c_{i}, D_{i}$ such that $c_{i} D_{i} \equiv c_{0} D_{0}(A), a \downarrow_{A} \bigcup_{i \leq n} D_{i}, t\left(c_{i} / D_{i}\right) \not \perp q_{0}$ and $a \in \operatorname{acl}\left(\bigcup_{i \leq n} c_{i} D_{i} A\right)$.

Received by the editors December 26, 1985 and, in revised form, April 16, 1987.

1980 Mathematics Subject Classification (1985 Revision). Primary 03C45.

The writing of this paper was supported by an NSF Postdoctoral Research Fellowship. 
(Notice that semiminimality is a property of $\operatorname{stp}(a / A)$.) The relevant result is

THEOREM 0.3 (IN Ceq) [Sh, $\mathrm{V}, 4.11(2)]$. If $t(a / A)$ is nonorthogonal to the minimal type $q$, then there is a $b \in \operatorname{acl}(a A) \backslash \operatorname{acl}(A)$ such that $\operatorname{stp}(b / A)$ is semiminimal w.r.t. q.

Observe that Theorem 0.1 is this theorem in the special case when we can choose $q \in S(A)$. So, Theorem 0.3 is a kind of general coordinatization theorem. The natural questions to ask are

(1) Can we choose $q$ to be in $S(A)$ ?

(2) If we cannot choose $q \in S(A)$ does $q$ have some other nice property?

Theorem 0.3 is of only minor interest unless it can be applied to each type in the theory, as is the case with Coordinatization Theorem 0.1 in $\omega$-categorical, $\omega$-stable theories. By [L1, Propositions 4 and 7 ] a type is nonorthogonal to a minimal type in $T^{\mathrm{eq}}$ if (and only if) it has successor $U$-rank. Thus, Theorem 0.3 can be applied to every type in $T$ only if they all have finite $U$-rank. For the remainder of the section we let $T$ denote such a theory and we write $T$ for $T^{\mathrm{eq}}$.

We will say that $T$ has a good coordinatization theorem if the answer to (1) is affirmative. So, $\omega$-categorical, $\omega$-stable theories have a good coordinatization theorem. In [B1] we showed that if every minimal type in $T$ is locally modular, then $T$ has a good coordinatization theorem. Thus, "geometrical simplicity" seems to imply that there is a good coordinatization theorem. Indeed, it is not difficult to show that a good coordinatization theorem is equivalent to $T$ being 1-based. In [B3] we argued that geometrical complexity only occurs in an " $\omega$-stable part" of the theory. So, we expect that a failure of a good coordinatization theorem implies that $q$ is strongly minimal. Proving this is the major step in proving Theorem A.

This completes our motivation of the usefulness of Theorem A and why we call it a coordinatization theorem. Its proof is outlined at the beginning of $\S 2$. At this point we would like to discuss the connections with [CI], largely to answer the question: Why is this paper easier than, and independent from, its predecessor? There are two reasons. The first is that we were not looking for theorems like Theorem A in [CI]. There, we wished to analyze the geometrical aspects of arbitrary forking extensions of such a type. We defined an incidence geometry, the coordinate geometry, which we felt best reflected the interactions of the conjugates of this forking extension. Since it was unclear at the time what properties of the geometry would be most useful we simply proceeded to obtain as much information about it as we could. Experience now shows that the crucial result is Theorem A. We need only the geometrical properties of a maximal forking extension which allows us to prove this result. As we will see in $\S 2$ we do not need the full coordinate geometry to obtain these properties.

The second reason this paper is easier than $[\mathrm{CI}]$ is that Corollary 1.6 allows us to abandon the coordinate geometry. It, with minor input from Proposition 1.9, allows us to reduce Theorem A to finding a minimal type which is nonorthogonal to the original type, and not locally modular. This fact is deduced from rather elementary geometrical properties of the conjugates of the forking extension. (See the further motivation in §2.) Neither of the results, Lemmas 1.2 and 1.5, should be minimized. Without either of them we would have to analyze the coordinate geometry itself to obtain any result like Theorem A. 
What about a generalization of Theorem 0.2 ? Theorem 0.2 was obtained rather easily from Theorem 0.1 because the equivalence relation $\sim$ was obviously definable. This is not the case in general. In Proposition 1.9(ii) we show how to obtain a $c \in \operatorname{dcl}(a A)$ which serves the same purpose as $a / \sim$. There is, however, a loss in wanting a $c$ in $\operatorname{dcl}(a A)$, rather than just in $\operatorname{acl}(a A)$. In attempting to prove that $\operatorname{stp}(c / A)$ is semiminimal we get all of the properties of the definition except the requirement that all of the conjugate minimal types be nonorthogonal. (This deficiency also occurs in Coordinatization Theorem 0.2.) In $\S 1$ we discuss this relation, called $(q, A)$-algebraic, in detail.

1. Preliminaries. We assume a basic knowledge of stability theory as found in $[\mathbf{M}, \mathbf{P}$ or $\mathbf{S h}]$. For the most part our notation follows $[\mathbf{M}]$. (See $[\mathbf{C I}]$ for a detailed description of our notational conventions.)

We will make heavy use of Shelah's notion of a canonical base [Sh, III, 6.10] and the results in [B1] on restricted canonical bases. We refer the reader to [Sh or B1] for a summary of the canonical base technology. Let $p$ be a strong type (i.e., for some $a$ and $A, p=\operatorname{stp}(a / A)), C=C b(p)$. Assuming that $T$ is superstable there is a finite $c \subset C$ such that $C \subset \operatorname{acl}(c)$ by [Sh, III, 6.10]. Any such $c$ is called a restricted canonical base of $p$. If $q=p \mid C$ we call $q\lceil c$ a restricted canonical type. The most useful property of such types is their thriftiness. (That is, suppose that $t(a / c)$ is a restricted canonical type, $c^{\prime} \equiv c(a)$ and $a \bigcup_{c} c^{\prime}$. Then $c^{\prime} \in \operatorname{acl}(c)$.)

A possibly incomplete type $p$ is weakly minimal if $R(p, L, \infty)=1$. That is, there is a $\varphi \in p$ such that $\varphi \in q \Rightarrow U(q) \leq 1$, where $U$ denotes Lascar's foundation rank. A type is minimal if it is stationary and has $U$-rank 1 . Certainly, a stationary weakly minimal type is minimal. A minimal type $p$ will also be weakly minimal if there is a $\varphi \in p$ such that every stationary nonalgebraic $q$ containing $\varphi$ is minimal. The following are easy consequences of Theorems 5.6 and 5.8 in $[\mathbf{L}]$.

LEMMA 1.1. (i) Suppose that there is an $n<\omega$ such that $U(b / A)=U(b / A c)+$ n. Then $U(c / A)=U(c / A b)+n$.

(ii) If $U(b / A)<\omega$ then $U(b c / A)=U(c / A b)+U(b / A)$.

For the definition and basic properties of local modularity (as a property of minimal types) we refer the reader to $[\mathbf{B 2}, \S 1$ or B3, Appendix]. The reader need not understand the proofs in [B2] if he is willing to accept the main theorem.

LEMMA 1.2. Suppose that $p$ is stationary and weakly minimal. Then $p$ is locally modular or strongly minimal.

We will also make use of

LEMMA 1.3 [B1, 4.4]. Suppose that $q \in S(B)$ is a modular minimal type. Then for all $X, Y, Y^{\prime} \subset q(C)$, if $Y \equiv Y^{\prime}(X B)$ and $Y \uplus_{B} X, Y \uplus_{B} Y^{\prime}$.

The next easy lemma will come in handy when applying Lemma 1.3. Since a locally modular type is modular over a single realization we have

LEMMA 1.4. Suppose that $T$ is superstable, $M$ is an a-model, and $q \in S(M)$ is minimal and locally modular. Then $q$ is modular.

Since we may be dealing with minimal types which are not weakly minimal the next result is crucial. A regular type $p \in S(A)$ is trivial if $\{a\} \cup B \subset p(C)$ and $a \uplus_{A} B \Rightarrow a \uplus_{A} b$, for some $b \in B$. 
LEMMA 1.5 [B4, THEOREM A]. Suppose that $T$ is superstable and $q \in S(A)$ is nontrivial and has U-rank 1. Then $q$ is weakly minimal.

Combining Lemmas 1.2 and 1.5 we obtain

COROLlaRY 1.6. Suppose that $T$ is superstable and $p$ is minimal. Then $p$ is locally modular or strongly minimal.

We let $R M(-)$ denote Morley rank.

$(q, C)$-algebraicity

DEFINITION. Let $A$ and $B_{0}$ be sets, $p$ and $q_{0}$ possibly incomplete types over $\operatorname{acl}(A)$ and $B_{0}$, respectively. Let $C \subset A$. We say that $p$ is $\left(q_{0}, C\right)$-algebraic if there is an $n<\omega, B_{i} \equiv B_{0}(C)$ (for $i \leq n$ ) and some finite set $D$ such that (letting $q_{i}$ denote $\left.q_{B_{i}}\right)$ : whenever $a$ realizes $p$ and $a \downarrow_{A} \bigcup_{i \leq n} B_{i} \cup D, a \in \operatorname{acl}\left(\bigcup_{i \leq n} q_{i}(\mathcal{C}) \cup B_{i} \cup A \cup D\right)$. If $A=C$ we say $q_{0}$-algebraic.

REMARK. Suppose that we have $a$ and $q_{i}(i \leq n)$ as above witnessing that $\operatorname{stp}(a / A)$ is $q_{0}$-algebraic. Further suppose that $q_{0}$ is minimal and $q_{0} \not \subset q_{i}$ for all $i \leq n$. Then $\operatorname{stp}(a / A)$ is $q_{0}$-semiminimal.

In $[\mathbf{H r}]$ we find the more general concepts: $p$-simple (for $p$ a regular type) and $P$-internal (for $P$ a family of types). Since $p$-simplicity is of interest largely when $p$ is not minimal we will not discuss it here. The reader is invited to notice that $(q, C)$-algebraicity is very close to being $P$-internal for $P$ the family of $C$-conjugates of $q$.

LEMMA 1.7. If a type $p$ is $(q, C)$-algebraic and $q$ has Morley rank 1 , then $p$ has finite Morley rank.

PROOF. Suppose that $p$ is a type over $A$. It suffices to show that an arbitrary completion $r$ of $p$ over $A$ has finite Morley rank. The hypotheses say that there are a realizing $r$, a finite $D$ and $\bar{b}=b_{0} \cdots b_{n}$ such that $R M\left(b_{i} / D\right)=1(i \leq n), a \downarrow_{A} D$ and $a \in \operatorname{acl}(D \bar{b})$. It follows that $R M(a / D A) \leq R M(a \bar{b} / A D)<\omega$. Since $a \downarrow_{A} D$, $R M(a / A)=R M(r)<\omega$.

The next lemma will not be used explicitly, but it does help to clarify our picture of the $q$-algebraic relation.

LEMMA 1.8. Suppose that $p$ is a type over acl $(A)$ which is $(q, C)$-algebraic for some $C \subset A$ and $q$ is strongly minimal. Then there are: a finite $E, q_{i}$ over $D_{i}$ with $q_{i}$ conjugate over $C$ to $q(i \leq n)$ such that $p(C) \subset \operatorname{acl}\left(\bigcup_{i \leq n} q_{i}(C) \cup D_{i} \cup E \cup A\right)$.

PROOF. This is a straightforward proof using weight. It is virtually identical to the proof of Lemma 3.10 in $[\mathbf{C I}]$.

We come now to the major existence theorem concerning semiminimal and $q$ algebraic types. The first part is just Theorem 0.3 , but we include a proof for completeness.

Proposition 1.9. Let $T^{\mathrm{eq}}$ be superstable. Suppose that $a, A$ and $d$ are such that there is a minimal type $q \in S(d)$ with $\operatorname{stp}(a / A) \not \perp q$. Then

(i) There is a $b \in \operatorname{acl}(a A) \backslash \operatorname{acl}(A)$ such that $\operatorname{stp}(b / A)$ is q-semiminimal.

(ii) There is a $b \in \operatorname{dcl}(a A) \backslash \operatorname{acl}(A)$ such that $\operatorname{stp}(b / A)$ is q-algebraic.

PROOF. Wlog, we may assume that $A=\phi, a \uplus d$, and $d$ is large enough so that there is a $c$ realizing $q$ with $a \uplus_{d} \quad c$. Let $J=\left\{c_{i} d_{i}: i<\omega\right\}$ be a Morley sequence 
in $\operatorname{stp}(c d / a)$. Let $b$ be a restricted canonical base of this strong type. Then $b \in$ $\operatorname{acl}(a) \cap \operatorname{acl}(\bigcup J) . J$ is not independent since $c_{i} \uplus_{a} d_{i}$, so $b \notin \operatorname{acl}(\phi)$. The assumption that $d \Psi a$ implies that $\{a\} \cup\left\{d_{i}: i<\omega\right\}$ is independent. Thus, $b 山 \cup\left\{d_{i}: i<\omega\right\}$. It follows immediately that $\operatorname{stp}(b)$ is $q$-algebraic since $t\left(c_{i} / d_{i}\right)=q_{d_{i}}$. Since $d_{i} \stackrel{s}{=} d_{j}$ and $d_{i} \Psi d_{j}$ for all $i, j$, and since $q \not \mathcal{\phi}$, all of the $q_{d_{i}}$ 's are nonorthogonal. Thus, $\operatorname{stp}(b)$ is $q$-semiminimal. This proves (i).

To prove (ii) first let $b$ be as obtained in (i). Let $\left\{b_{0}, \ldots, b_{n}\right\}$ be the set of realizations of $t(b / a), b^{\prime}$ a name for this set in $C^{\text {eq }}$. Then $b^{\prime} \in \operatorname{dcl}(a)$. For each $i$, $\operatorname{stp}\left(b_{i}\right)$ is semiminimal with respect to some conjugate of $q$. It follows easily that $\operatorname{stp}\left(b^{\prime}\right)$ is $q$-algebraic.

2. Proof of Theorem A. Throughout the section $T$ denotes $T^{\text {eq }}$ of a superstable theory, $a, A$ and $\alpha$ are as in the statement of Theorem A. Wlog, $A=\varnothing$. First we will motivate and outline the proof.

Find a $b$ such that $U(a / b)=\alpha$ and $t(a / b)$ is a restricted canonical type. If $U(b)=1$ then $b \in \operatorname{acl}(a)$ and we may set $c=b$ to satisfy (i) of Theorem A. So, assume that $U(b)>1$. This will yield a $b^{\prime}$ such that $b^{\prime} \stackrel{s}{=} b(a), b^{\prime} \uplus_{a} \quad b$ and $b^{\prime} \Downarrow b$. From experience we know that this configuration gives rise to a set of $U$-rank 1, nonorthogonal to $\operatorname{stp}(a)$, which is not locally modular. By Corollary 1.6 we have a strongly minimal formula $\theta$ nonorthogonal to $\operatorname{stp}(a)$. Thus, by Proposition 1.9 there is a $c \in \operatorname{acl}(a) \backslash \operatorname{acl}(\phi)$ such that $\operatorname{stp}(c)$ is $\theta$-semiminimal. By Lemma 1.7, $\operatorname{stp}(c)$ has finite Morley rank, proving (ii).

Now we proceed with the details of the proof.

LEMMA 2.1. $U(b)<\omega$.

ProOF. Let $\bar{d}$ be a finite Morley sequence in $\operatorname{stp}(a / b)$ such that this strong type is based on $\bar{d}$. Since we chose $b$ so that $U(a / b)+1=U(a)$ and since $\bar{d}$ is a Morley sequence in this strong type, we can induct on $\ln (\bar{d})$ to see that there is a $k<\omega$ such that $U(\bar{d} / b)+k=U(\bar{d})$ by an easy application of $[\mathbf{L}$, Theorem 8]: $U(e f / B) \leq U(e / B f) \oplus U(f / B)$. Since $b$ is in the canonical base of $\operatorname{stp}(a / b)$, $b \in \operatorname{acl}(\bar{d})$. By Lemma $1.1(\mathrm{i}), U(b)=k$.

If $k=U(b)=1$, as in the intuitive outline we set $c=b$ and Theorem A (i) holds. So, suppose that $U(b)>1$. By Lemma 1.1 (i)

$$
0<U(b / a)=U(b)-1 \text {. }
$$

Our first goal is to find a $b^{\prime}$ relating to $a$ and $b$ as in the outline. Choose $b^{\prime}$ such that

$$
b^{\prime} \stackrel{s}{=} b(a) \quad \text { and } \quad b^{\prime} \uplus_{a} \quad b
$$

LEMMA 2.2. $b^{\prime} \downarrow b$.

Proof. Suppose, towards a contradiction, that $b^{\prime} \Downarrow b$. By (1) and (2) we have $U\left(b^{\prime}\right)-1=U\left(b^{\prime} / a b\right) \leq U\left(b^{\prime} / b\right)<U\left(b^{\prime}\right)$. Thus, we must have $U\left(b^{\prime} / a b\right)=U\left(b^{\prime} / b\right)$, i.e., $b^{\prime} \Psi_{b} a$. Since $t(a / b)$ is a restricted canonical type, $b^{\prime} \in \operatorname{acl}(b)$. This contradicts (1) and (2).

We will now show that this configuration of $a, b$ and $b^{\prime}$ leads to a minimal type which is nonorthogonal to $\operatorname{stp}(a)$ and not locally modular. We first use regular 
decompositions [M, D.10] to transfer this configuration into a minimal set. Then we apply Lemma 1.3 to conclude that this type is not locally modular.

Let $M$ be a large saturated model, $M \uplus a b b^{\prime}$. Notice that

$$
b^{\prime} \stackrel{s}{\equiv} b(a M), \quad b^{\prime}{\underset{a M}{\|}}_{b} b \quad \text { and } \quad b^{\prime} \underset{M}{\uplus_{M}} b .
$$

Let $\bar{d}$ be a regular decomposition of $b$ over $M$. That is, $\bar{d}$ is $M$-independent, $e \in \bar{d} \Rightarrow t(e / M)$ is regular, $b \triangleleft_{M} \bar{d}$ and $\bar{d} \triangleleft_{M} b$. By [L1, Propositions 4 and 7] every regular type nonorthogonal to $t(b / M)$ is nonorthogonal to a minimal type. Thus, wlog, $e \in \bar{d} \Rightarrow t(e / M)$ is minimal. Of course, this implies that $\bar{d} \in \operatorname{acl}(b M)$. Let $\bar{e}$ be a regular decomposition of $a$ over $M$. We can make the additional requirement that

$$
f, f^{\prime} \in \bar{d} \cup \bar{e} \text { and } t(f / M) \not \perp t\left(f^{\prime} / M\right) \text { implies that } f \equiv f^{\prime}(M) .
$$

Since $a \downarrow_{M} b, \bar{d} \downarrow_{M} \bar{e}$ ( $a$ and $b$ are dominated over $M$ by their decompositions). So, some element of the sequence $\bar{d}$ has a type nonorthogonal to $t(\bar{e} / M)$. It follows that there are $\bar{d}^{\prime} \subset \bar{d}$ and $\bar{e}^{\prime} \subset \bar{e}$ such that

(5) $\bar{d}^{\prime} \uplus_{M} \bar{e}^{\prime}$ and the elements of $\bar{d}^{\prime} \cup \bar{e}^{\prime}$ all have the same type $q$ over $M$.

Now find a $\bar{d}^{\prime \prime}$ such that $b^{\prime} \bar{d}^{\prime \prime} \stackrel{s}{\equiv} b \bar{d}^{\prime}(a M)$. By (3), $\bar{d}^{\prime \prime} \downarrow_{M} \bar{d}^{\prime}$. Since $\bar{e}^{\prime} \in \operatorname{acl}(a M)$, $\bar{d}^{\prime \prime} \stackrel{s}{\equiv} \bar{d}^{\prime}\left(\bar{e}^{\prime} M\right)$. So, by (5), Lemmas 1.3 and 1.4 we conclude that $q$ is not locally modular. Since $\bar{e}^{\prime} \neq \phi, q \not \mathcal{s t p}(a)$. $q$ is strongly minimal by Corollary 1.6.

We can now proceed as in the outline to conclude that there is a $c \in \operatorname{acl}(a) \operatorname{such}$ that $\operatorname{stp}(c)$ is $q$-semiminimal. This proves (ii) of Theorem A.

3. Concluding remarks. Applying Proposition 1.9 (ii) to the conclusion of Theorem A we obtain the following analogue to Coordinatization Theorem 0.2.

THEOREM 3.1. Suppose that $T$ is superstable and $U(a / A)=\alpha+1$, for some $\alpha$. Then in $T^{\mathrm{eq}}$ there is a $c \in \operatorname{dcl}(a A) \backslash \operatorname{acl}(A)$ such that one of the following holds.

(i) $U(c / A)=1$, or

(ii) $\operatorname{stp}(c / A)$ is $(\theta, A)$-algebraic for some strongly minimal formula $\theta$.

Since this research was originally completed another proof of Theorem $\mathrm{A}$ has become possible. Suppose that $a$ and $A$ are as in the statement. Then $\operatorname{stp}(a / A)$ is nonorthogonal to some minimal type $q$. If $q$ is locally modular we can apply Theorem 5.1 in $[\mathbf{H r}]$ to see that Theorem A (i) holds. (The reader needs to check that in Theorem 5.1, when $p$ is minimal, we can replace $\mathrm{Cl}_{p}(-)$ by $\operatorname{acl}(-)$.) So, now assume that $q$ is not locally modular. By Corollary 1.6 it is strongly minimal, so we can proceed as in $\S 2$ to obtain Theorem A (ii).

\section{REFERENCES}

[CI] S. Buechler, Coordinatization in superstable theories. I: Stationary types, Trans. Amer. Math. Soc. 288 (1985), 101-114.

[B1] _ Locally modular theories of finite rank, Ann. Pure Appl. Logic 30 (1986), 83-94.

[B2] _ The geometry of weakly minimal types, J. Symbolic Logic 50 (1985), 1044-1053.

[B3] _ Geometrical stability theory, Logic Colloquium '85, North-Holland, 1987, pp. 53-66.

[B4] _ Non-trivial types of U-rank 1, J. Symbolic Logic 52 (1987), 548-551.

[B5] _ Invariants in $\omega$-categorical, $\omega$-stable theories, Israel J. Math. 52 (1985), 65-81. 
[CHL] G. Cherlin, L. Harrington and A. H. Lachlan, $\aleph_{0}$-categorical, $\aleph_{0}$-stable structures, Ann. Pure Appl. Logic 28 (1985), 103-136.

[L] D. Lascar, Ranks and definability in superstable theories, Israel J. Math. 23 (1976).

[L1] __, Relation entre le rang $U$ et le poids, Fund. Math. 121 (1984), 117-123.

[LP] D. Lascar and B. Poizat, An introduction to forking, J. Symbolic Logic 44 (1979), 330-350.

[M] M. Makkai, A survey of basic stability theory, with particular emphasis on orthogonality and regular types, Israel J. Math. 49 (1984), 181-238.

[Sh] S. Shelah, Classification theory, North-Holland, 1978.

[A] G. Ahlbrandt, Doctoral dissertation, Univ. of Illinois at Chicago, 1984 (submitted).

[Hr] E. Hrushovski, Contributions to stable model theory, Doctoral Dissertation, Univ. of CaliforniaBerkeley, 1986 (submitted).

Department of Mathematics, University of Wisconsin-Milwaukee, MilwauKEE, WISCONSIN 53201 